\title{
On limiting values of Cauchy type integral in a harmonic algebra with two-dimensional radical
}

\begin{abstract}
We consider a certain analog of Cauchy type integral taking values in a three-dimensional harmonic algebra with two-dimensional radical. We establish sufficient conditions for an existence of limiting values of this integral on the curve of integration.
\end{abstract}

1. Introduction. Let $\Gamma$ be a closed Jordan rectifiable curve in the complex plane $\mathbb{C}$. By $D^{+}$and $D^{-}$we denote, respectively, the interior and the exterior domains bounded by the curve $\Gamma$.

N. Davydov [1] established sufficient conditions for an existence of limiting values of the Cauchy type integral

$$
\frac{1}{2 \pi i} \int_{\Gamma} \frac{g(t)}{t-\xi} d t, \quad \xi \in \mathbb{C} \backslash \Gamma,
$$

on $\Gamma$ from the domains $D^{+}$and $D^{-}$. This result stimulated a development of the theory of Cauchy type integral on curves which are not piecewisesmooth.

In particular, using the mentioned result of the paper [1], the following result was proved: if the curve $\Gamma$ satisfies the condition (see [2])

$$
\theta(\varepsilon):=\sup _{\xi \in \Gamma} \theta_{\xi}(\varepsilon)=O(\varepsilon), \quad \varepsilon \rightarrow 0
$$

2010 Mathematics Subject Classification. 30G35, 30E25.

Key words and phrases. Three-dimensional harmonic algebra, Cauchy type integral, limiting values, closed Jordan rectifiable curve. 
(here $\theta_{\xi}(\varepsilon):=$ mes $\{t \in \Gamma:|t-\xi| \leq \varepsilon\}$, where mes denotes the linear Lebesgue measure on $\Gamma$ ), and the modulus of continuity

$$
\omega_{g}(\varepsilon):=\sup _{t_{1}, t_{2} \in \Gamma,\left|t_{1}-t_{2}\right| \leq \varepsilon}\left|g\left(t_{1}\right)-g\left(t_{2}\right)\right|
$$

of a function $g: \Gamma \rightarrow \mathbb{C}$ satisfies the Dini condition

$$
\int_{0}^{1} \frac{\omega_{g}(\eta)}{\eta} d \eta<\infty,
$$

then the integral (1) has limiting values in every point of $\Gamma$ from the domains $D^{+}$and $D^{-}$(see [3]). The condition (2) means that the measure of a part of the curve $\Gamma$ in every disk centered at a point of the curve is commensurable with the radius of the disk.

In this paper we consider a certain analogue of Cauchy type integral taking values in a three-dimensional harmonic algebra with two-dimensional radical and study the question about an existence of its limiting values on the curve of integration.

\section{A three-dimensional harmonic algebra with a two-dimensional}

radical. Let $\mathbb{A}_{3}$ be a three-dimensional commutative associative Banach algebra with unit 1 over the field of complex numbers $\mathbb{C}$. Let $\left\{1, \rho_{1}, \rho_{2}\right\}$ be a basis of algebra $\mathbb{A}_{3}$ with the multiplication table: $\rho_{1} \rho_{2}=\rho_{2}^{2}=0, \rho_{1}^{2}=\rho_{2}$.

$\mathbb{A}_{3}$ is a harmonic algebra, i.e. there exists a harmonic basis $\left\{e_{1}, e_{2}, e_{3}\right\} \subset$ $\mathbb{A}_{3}$ satisfying the following conditions (see [5], [6], [7], [8], [9]):

$$
e_{1}^{2}+e_{2}^{2}+e_{3}^{2}=0, \quad e_{j}^{2} \neq 0 \text { for } j=1,2,3 .
$$

P. Ketchum [5] discovered that every function $\Phi(\zeta)$ analytic with respect to the variable $\zeta:=x e_{1}+y e_{2}+z e_{3}$ with real $x, y, z$ satisfies the equalities

$$
\left(\frac{\partial^{2}}{\partial x^{2}}+\frac{\partial^{2}}{\partial y^{2}}+\frac{\partial^{2}}{\partial z^{2}}\right) \Phi(\zeta)=\Phi^{\prime \prime}(\zeta)\left(e_{1}^{2}+e_{2}^{2}+e_{3}^{2}\right)=0
$$

owing to the equality (4). I. Mel'nichenko [7] noticed that doubly differentiable in the sense of Gateaux functions form the largest class of functions $\Phi$ satisfying the equalities (5).

All harmonic bases in $\mathbb{A}_{3}$ are constructed by I. Mel'nichenko in [9].

Consider a harmonic basis

$$
e_{1}=1, \quad e_{2}=i+\frac{1}{2} i \rho_{2}, \quad e_{3}=-\rho_{1}-\frac{\sqrt{3}}{2} i \rho_{2}
$$

in $\mathbb{A}_{3}$ and the linear envelope $E_{3}:=\left\{\zeta=x+y e_{2}+z e_{3}: x, y, z \in \mathbb{R}\right\}$ over the field of real numbers $\mathbb{R}$, that is generated by the vectors $1, e_{2}, e_{3}$. Associate with a domain $\Omega \subset \mathbb{R}^{3}$ the domain $\Omega_{\zeta}:=\left\{\zeta=x+y e_{2}+z e_{3}:(x, y, z) \in \Omega\right\}$ in $E_{3}$. 
The algebra $\mathbb{A}_{3}$ have the unique maximal ideal $\left\{\lambda_{1} \rho_{1}+\lambda_{2} \rho_{2}: \lambda_{1}, \lambda_{2} \in \mathbb{C}\right\}$ which is also the radical of $\mathbb{A}_{3}$. Thus, it is obvious that the straight line $\left\{z e_{3}: z \in \mathbb{R}\right\}$ is contained in the radical of algebra $\mathbb{A}_{3}$.

$\mathbb{A}_{3}$ is a Banach algebra with the Euclidean norm

$$
\|a\|:=\sqrt{\left|\xi_{1}\right|^{2}+\left|\xi_{2}\right|^{2}+\left|\xi_{3}\right|^{2}},
$$

where $a=\xi_{1}+\xi_{2} e_{2}+\xi_{3} e_{3}$ and $\xi_{1}, \xi_{2}, \xi_{3} \in \mathbb{C}$.

We say that a continuous function $\Phi: \Omega_{\zeta} \rightarrow \mathbb{A}_{3}$ is monogenic in a domain $\Omega_{\zeta} \subset E_{3}$ if $\Phi$ is differentiable in the sense of Gateaux in every point of $\Omega_{\zeta}$, i. e. if for every $\zeta \in \Omega_{\zeta}$ there exists $\Phi^{\prime}(\zeta) \in \mathbb{A}_{3}$ such that

$$
\lim _{\varepsilon \rightarrow 0+0}(\Phi(\zeta+\varepsilon h)-\Phi(\zeta)) \varepsilon^{-1}=h \Phi^{\prime}(\zeta) \quad \forall h \in E_{3} .
$$

For monogenic functions $\Phi: \Omega_{\zeta} \rightarrow \mathbb{A}_{3}$ we established basic properties analogous to properties of analytic functions of the complex variable: the Cauchy integral theorem, the Cauchy integral formula, the Morera theorem, the Taylor expansion (see [11]).

\section{On existence of limiting values of a hypercomplex analogue of} the Cauchy type integral. In what follows, $t_{1}, t_{2}, x, y, z \in \mathbb{R}$ and the variables $x, y, z$ with subscripts are real. For example, $x_{0}$ and $x_{1}$ are real, etc.

Let $\Gamma_{\zeta}:=\left\{\tau=t_{1}+t_{2} e_{2}: t_{1}+i t_{2} \in \Gamma\right\}$ be the curve congruent to the curve $\Gamma \subset \mathbb{C}$. Consider the domain $\Pi_{\zeta}^{ \pm}:=\left\{\zeta=x+y e_{2}+z e_{3}: x+i y \in D^{ \pm}, z \in \mathbb{R}\right\}$ in $E_{3}$. By $\Sigma_{\zeta}$ we denote the common boundary of domains $\Pi_{\zeta}^{+}$and $\Pi_{\zeta}^{-}$.

Consider the integral

$$
\Phi(\zeta)=\frac{1}{2 \pi i} \int_{\Gamma_{\zeta}} \varphi(\tau)(\tau-\zeta)^{-1} d \tau
$$

with a continuous density $\varphi: \Gamma_{\zeta} \rightarrow \mathbb{R}$. The function (6) is monogenic in the domains $\Pi_{\zeta}^{+}$and $\Pi_{\zeta}^{-}$, but the integral (6) is not defined for $\zeta \in \Sigma_{\zeta}$.

For the function $\varphi: \Gamma_{\zeta} \rightarrow \mathbb{R}$ consider the modulus of continuity

$$
\omega_{\varphi}(\varepsilon):=\sup _{\tau_{1}, \tau_{2} \in \Gamma_{\zeta},\left\|\tau_{1}-\tau_{2}\right\| \leq \varepsilon}\left|\varphi\left(\tau_{1}\right)-\varphi\left(\tau_{2}\right)\right|,
$$

and a singular integral

$$
\int_{\Gamma_{\zeta}}\left(\varphi(\tau)-\varphi\left(\zeta_{0}\right)\right)\left(\tau-\zeta_{0}\right)^{-1} d \tau:=\lim _{\varepsilon \rightarrow 0} \int_{\Gamma_{\zeta} \backslash \Gamma_{\zeta}^{\varepsilon}\left(\zeta_{0}\right)}\left(\varphi(\tau)-\varphi\left(\zeta_{0}\right)\right)\left(\tau-\zeta_{0}\right)^{-1} d \tau,
$$

where $\zeta_{0} \in \Gamma_{\zeta}$ and $\Gamma_{\zeta}^{\varepsilon}\left(\zeta_{0}\right):=\left\{\tau \in \Gamma_{\zeta}:\left\|\tau-\zeta_{0}\right\| \leq \varepsilon\right\}$.

Below, in Theorem 1 in the case where the curve $\Gamma$ satisfies the condition (2) and the modulus of continuity of the function $\varphi$ satisfies a condition of the type (3), we establish the existence of certain limiting values of the integral (6) in points $\zeta_{0} \in \Gamma_{\zeta}$ when $\zeta$ tends to $\zeta_{0}$ from $\Pi_{\zeta}^{+}$or $\Pi_{\zeta}^{-}$along 
a curve that is not tangential to the surface $\Sigma_{\zeta}$ outside of the plane of curve $\Gamma_{\zeta}$.

For the Euclidean norm in $\mathbb{A}_{3}$ the following inequalities are fulfilled:

$$
\begin{gathered}
\|a b\| \leq 2 \sqrt{14}\|a\|\|b\| \quad \forall a, b \in \mathbb{A}_{3}, \\
\left\|\int_{\Gamma_{\zeta}^{\prime}} \psi(\tau) d \tau\right\| \leq 9 M \int_{\Gamma_{\zeta}^{\prime}}\|\psi(\tau)\|\|d \tau\|
\end{gathered}
$$

with the constant $M:=\max \left\{1,\left\|e_{2}^{2}\right\|,\left\|e_{2} e_{3}\right\|,\left\|e_{3}^{2}\right\|\right\}$ for any measurable set $\Gamma_{\zeta}^{\prime} \subset \Gamma_{\zeta}$ and all continuous functions $\psi: \Gamma_{\zeta}^{\prime} \rightarrow \mathbb{A}_{3}$.

Lemma 1. Let $\Gamma$ be a closed Jordan rectifiable curve satisfying the condition (2) and the modulus of continuity of a function $\varphi: \Gamma_{\zeta} \rightarrow \mathbb{R}$ satisfies the condition of the type (3). If a point $\zeta$ tends to $\zeta_{0} \in \Gamma_{\zeta}$ along a curve $\gamma_{\zeta}$ for which there exists a constant $m<1$ such that the inequality

$$
|z| \leq m\left\|\zeta-\zeta_{0}\right\|
$$

is fulfilled for all $\zeta=x+y e_{2}+z e_{3} \in \gamma_{\zeta}$, then

$$
\lim _{\zeta \rightarrow \zeta_{0}, \zeta \in \gamma_{\zeta}} \int_{\Gamma_{\zeta}}\left(\varphi(\tau)-\varphi\left(\zeta_{0}\right)\right)(\tau-\zeta)^{-1} d \tau=\int_{\Gamma_{\zeta}}\left(\varphi(\tau)-\varphi\left(\zeta_{0}\right)\right)\left(\tau-\zeta_{0}\right)^{-1} d \tau .
$$

Proof. Let $\varepsilon:=\left\|\zeta-\zeta_{0}\right\|$. Consider the difference

$$
\begin{aligned}
& \int_{\Gamma_{\zeta}}\left(\varphi(\tau)-\varphi\left(\zeta_{0}\right)\right)(\tau-\zeta)^{-1} d \tau-\int_{\Gamma_{\zeta}}\left(\varphi(\tau)-\varphi\left(\zeta_{0}\right)\right)\left(\tau-\zeta_{0}\right)^{-1} d \tau \\
& =\int_{\Gamma_{\zeta}^{2 \varepsilon}\left(\zeta_{0}\right)}\left(\varphi(\tau)-\varphi\left(\zeta_{0}\right)\right)(\tau-\zeta)^{-1} d \tau-\int_{\Gamma_{\zeta}^{2 \varepsilon}\left(\zeta_{0}\right)}\left(\varphi(\tau)-\varphi\left(\zeta_{0}\right)\right)\left(\tau-\zeta_{0}\right)^{-1} d \tau \\
& +\left(\zeta-\zeta_{0}\right) \int_{\Gamma_{\zeta} \backslash \Gamma_{\zeta}^{2 \varepsilon}\left(\zeta_{0}\right)}\left(\varphi(\tau)-\varphi\left(\zeta_{0}\right)\right)(\tau-\zeta)^{-1}\left(\tau-\zeta_{0}\right)^{-1} d \tau=: I_{1}-I_{2}+I_{3} .
\end{aligned}
$$

To estimate $I_{1}$ we choose a point $\zeta_{1}=x_{1}+y_{1} e_{2}$ on $\Gamma_{\zeta}$ such that $\left\|\zeta-\zeta_{1}\right\|=$ $\min _{\tau \in \Gamma_{\zeta}}\|\tau-\zeta\|$. Using the inequalities (7) and (8), we obtain

$$
\begin{gathered}
\left\|I_{1}\right\|=\left\|\int_{\Gamma_{\zeta}^{2 \varepsilon}\left(\zeta_{0}\right)}\left(\varphi(\tau)-\varphi\left(\zeta_{1}\right)\right)(\tau-\zeta)^{-1} d \tau+\left(\varphi\left(\zeta_{1}\right)-\varphi\left(\zeta_{0}\right)\right) \int_{\Gamma_{\zeta}^{2 \varepsilon}\left(\zeta_{0}\right)}(\tau-\zeta)^{-1} d \tau\right\| \\
\leq 18 \sqrt{14} M \int_{\Gamma_{\zeta}^{2 \varepsilon}\left(\zeta_{0}\right)}\left|\varphi(\tau)-\varphi\left(\zeta_{1}\right)\right|\left\|(\tau-\zeta)^{-1}\right\|\|d \tau\|
\end{gathered}
$$




$$
+\left|\varphi\left(\zeta_{1}\right)-\varphi\left(\zeta_{0}\right)\right|\left\|\int_{\Gamma_{\zeta}^{2 \varepsilon}\left(\zeta_{0}\right)}(\tau-\zeta)^{-1} d \tau\right\|=: I_{1}^{\prime}+I_{1}^{\prime \prime}
$$

It follows from Lemma $1.1[9]$ that

$$
(\tau-\zeta)^{-1}=\frac{1}{t-\xi}-\frac{z}{(t-\xi)^{2}} \rho_{1}+\left(\frac{i}{2} \frac{y-t_{2}-\sqrt{3} z}{(t-\xi)^{2}}+\frac{z^{2}}{(t-\xi)^{3}}\right) \rho_{2}
$$

for all $\zeta=x+y e_{2}+z e_{3} \in \Pi_{\zeta}^{ \pm}$and $\tau=t_{1}+t_{2} e_{2} \in \Gamma_{\zeta}$, where $\xi:=x+i y$ and $t:=t_{1}+i t_{2}$. The following inequality follows from the relations (9) and (10):

$$
\left\|(\tau-\zeta)^{-1}\right\| \leq c(m) \frac{1}{|t-\xi|},
$$

where the constant $c(m)$ depends only on $m$.

Using the inequality $|t-\xi| \geq\left|t-\xi_{1}\right| / 2$ with $\xi_{1}:=x_{1}+i y_{1}$ and the inequality (11), we obtain:

$$
\begin{aligned}
\left\|I_{1}^{\prime}\right\| & \leq 18 \sqrt{14} M c(m) \int_{\Gamma_{\zeta}^{2 \varepsilon}\left(\zeta_{0}\right)} \frac{\left|\varphi(\tau)-\varphi\left(\zeta_{1}\right)\right|}{|t-\xi|}\|d \tau\| \\
& \leq 36 \sqrt{14} M c(m) \int_{\Gamma_{\zeta}^{2 \varepsilon}\left(\zeta_{0}\right)} \frac{\left|\varphi(\tau)-\varphi\left(\zeta_{1}\right)\right|}{\left|t-\xi_{1}\right|}\|d \tau\| \\
& \leq 36 \sqrt{14} M c(m) \int_{[0,4 \varepsilon]} \frac{\omega_{\varphi}(\eta)}{\eta} d \theta_{\xi_{1}}(\eta),
\end{aligned}
$$

where the last integral is understood as a Lebesgue-Stieltjes integral.

To estimate the last integral we use Proposition 1 [10] (see also the proof of Theorem 1 [4]) and the condition (2). So, we have

$$
\int_{[0,4 \varepsilon]} \frac{\omega_{\varphi}(\eta)}{\eta} d \theta_{\xi_{1}}(\eta) \leq \int_{0}^{8 \varepsilon} \frac{\theta_{\xi_{1}}(\eta) \omega_{\varphi}(\eta)}{\eta^{2}} d \eta \leq c \int_{0}^{8 \varepsilon} \frac{\omega_{\varphi}(\eta)}{\eta} d \eta \rightarrow 0, \quad \varepsilon \rightarrow 0,
$$

where the constant $c$ does not depend on $\varepsilon$.

To estimate $I_{1}^{\prime \prime}$ we introduce the domain $D_{\zeta}^{2 \varepsilon}\left(\zeta_{0}\right):=\left\{\tau=t_{1}+t_{2} e_{2}\right.$ : $\left.t_{1}+i t_{2} \in D^{+},\left\|\tau-\zeta_{0}\right\| \leq 2 \varepsilon\right\}$ and its boundary $\partial D_{\zeta}^{2 \varepsilon}\left(\zeta_{0}\right)$. Using the inequalities (8) and (11), we obtain: 


$$
\begin{aligned}
\left\|I_{1}^{\prime \prime}\right\| & \leq \omega_{\varphi}\left(\left\|\zeta_{1}-\zeta_{0}\right\|\right)\left\|\int_{\Gamma_{\zeta}^{2 \varepsilon}\left(\zeta_{0}\right)}(\tau-\zeta)^{-1} d \tau\right\| \\
& =\omega_{\varphi}\left(\left\|\zeta_{1}-\zeta_{0}\right\|\right)\left\|\int_{\partial D_{\zeta}^{2 \varepsilon}\left(\zeta_{0}\right)}(\tau-\zeta)^{-1} d \tau-\int_{\partial D_{\zeta}^{2 \varepsilon}\left(\zeta_{0}\right) \backslash \Gamma_{\zeta}^{2 \varepsilon}\left(\zeta_{0}\right)}(\tau-\zeta)^{-1} d \tau\right\| \\
& \leq \omega_{\varphi}\left(\left\|\zeta_{1}-\zeta_{0}\right\|\right)\left(2 \pi+9 M c(m) \int_{\partial D_{\zeta}^{2 \varepsilon}\left(\zeta_{0}\right) \backslash \Gamma_{\zeta}^{2 \varepsilon}\left(\zeta_{0}\right)} \frac{\|d \tau\|}{|t-\xi|}\right) \\
& \leq \omega_{\varphi}(2 \varepsilon)\left(2 \pi+9 M c(m) \frac{1}{\varepsilon} 2 \pi 2 \varepsilon\right) \rightarrow 0, \quad \varepsilon \rightarrow 0 .
\end{aligned}
$$

Estimating $I_{2}$, by analogy with the estimation of $I_{1}^{\prime}$, we obtain:

$$
\left\|I_{2}\right\| \leq c \int_{0}^{4 \varepsilon} \frac{\omega_{\varphi}(\eta)}{\eta} d \eta \rightarrow 0, \quad \varepsilon \rightarrow 0,
$$

where the constant $c$ does not depend on $\varepsilon$.

Using the inequality $|t-\xi| \geq\left|t-\xi_{0}\right| / 2$, where the point $\xi_{0}:=x_{0}+i y_{0}$ corresponds to the point $\zeta_{0}=x_{0}+y_{0} e_{2}$, and using the relations (7), (8), (11) and (2), by analogy with the estimation of $I_{1}^{\prime}$, we obtain:

$$
\begin{aligned}
\left\|I_{3}\right\| & \leq 9 M(2 \sqrt{14})^{2} \varepsilon \int_{\Gamma_{\zeta} \backslash \Gamma_{\zeta}^{2 \varepsilon}\left(\zeta_{0}\right)}\left|\varphi(\tau)-\varphi\left(\zeta_{0}\right)\right|\left\|(\tau-\zeta)^{-1}\right\|\left\|\left(\tau-\zeta_{0}\right)^{-1}\right\|\|d \tau\| \\
& \leq c \varepsilon \int_{\Gamma_{\zeta} \backslash \Gamma_{\zeta}^{2 \varepsilon}\left(\zeta_{0}\right)} \frac{\left|\varphi(\tau)-\varphi\left(\zeta_{0}\right)\right|}{|t-\xi|\left|t-\xi_{0}\right|}\|d \tau\| \leq c \varepsilon \int_{\Gamma_{\zeta} \backslash \Gamma_{\zeta}^{2 \varepsilon}\left(\zeta_{0}\right)} \frac{\left|\varphi(\tau)-\varphi\left(\zeta_{0}\right)\right|}{\left|t-\xi_{0}\right|^{2}}\|d \tau\| \\
& \leq c \varepsilon \int_{[2 \varepsilon, d]} \frac{\omega_{\varphi}(\eta)}{\eta^{2}} d \theta_{\xi_{0}}(\eta) \leq c \varepsilon \int_{2 \varepsilon}^{2 d} \frac{\theta_{\xi_{0}}(\eta) \omega_{\varphi}(\eta)}{\eta^{3}} d \eta \\
& \leq c \varepsilon \int_{2 \varepsilon}^{2 d} \frac{\omega_{\varphi}(\eta)}{\eta^{2}} d \eta \rightarrow 0, \quad \varepsilon \rightarrow 0,
\end{aligned}
$$

where $d:=\max _{\xi_{1}, \xi_{2} \in \Gamma}\left|\xi_{1}-\xi_{2}\right|$ is the diameter of $\Gamma$ and $c$ denotes different constants which do not depend on $\varepsilon$. The lemma is proved.

Let $\widehat{\Phi}^{ \pm}\left(\zeta_{0}\right)$ be the boundary value of function (6) when $\zeta$ tends to $\zeta_{0} \in \Gamma_{\zeta}$ along a curve $\gamma_{\zeta}$ for which there exists a constant $m<1$ such that the inequality (9) is fulfilled for all $\zeta=x+y e_{2}+z e_{3} \in \gamma_{\zeta}$. 
Theorem 1. Let $\Gamma$ be a closed Jordan rectifiable curve satisfying the condition (2) and the modulus of continuity of a function $\varphi: \Gamma_{\zeta} \rightarrow \mathbb{R}$ satisfies the condition of the type (3). Then the integral (6) has boundary values $\widehat{\Phi}^{ \pm}\left(\zeta_{0}\right)$ for all $\zeta_{0} \in \Gamma_{\zeta}$ that are expressed by the formulas:

$$
\begin{gathered}
\widehat{\Phi}^{+}\left(\zeta_{0}\right)=\frac{1}{2 \pi i} \int_{\Gamma_{\zeta}}\left(\varphi(\tau)-\varphi\left(\zeta_{0}\right)\right)\left(\tau-\zeta_{0}\right)^{-1} d \tau+\varphi\left(\zeta_{0}\right) \\
\widehat{\Phi}^{-}\left(\zeta_{0}\right)=\frac{1}{2 \pi i} \int_{\Gamma_{\zeta}}\left(\varphi(\tau)-\varphi\left(\zeta_{0}\right)\right)\left(\tau-\zeta_{0}\right)^{-1} d \tau .
\end{gathered}
$$

The theorem follows from the Lemma 1 and the equalities

$$
\begin{gathered}
\frac{1}{2 \pi i} \int_{\Gamma_{\zeta}} \varphi(\tau)(\tau-\zeta)^{-1} d \tau=\frac{1}{2 \pi i} \int_{\Gamma_{\zeta}}\left(\varphi(\tau)-\varphi\left(\zeta_{0}\right)\right)(\tau-\zeta)^{-1} d \tau+\varphi\left(\zeta_{0}\right) \quad \forall \zeta \in \Pi_{\zeta}^{+}, \\
\frac{1}{2 \pi i} \int_{\Gamma_{\zeta}} \varphi(\tau)(\tau-\zeta)^{-1} d \tau=\frac{1}{2 \pi i} \int_{\Gamma_{\zeta}}\left(\varphi(\tau)-\varphi\left(\zeta_{0}\right)\right)(\tau-\zeta)^{-1} d \tau \quad \forall \zeta \in \Pi_{\zeta}^{-} .
\end{gathered}
$$

In comparison with Theorem 1, note that additional assumptions about the function $\varphi$ are required for an existence of limiting values of the function (6) from $\Pi_{\zeta}^{+}$or $\Pi_{\zeta}^{-}$on the boundary $\Sigma_{\zeta}$. We are going to state these results in next papers.

\section{REFERENCES}

[1] Davydov, N. A., The continuity of an integral of Cauchy type in a closed domain, Dokl. Akad. Nauk SSSR 64, no. 6 (1949), 759-762 (Russian).

[2] Salaev, V. V., Direct and inverse estimates for a singular Cauchy integral along a closed curve, Mat. Zametki 19, no. 3 (1976), 365-380 (Russian).

[3] Gerus, O. F., Finite-dimensional smoothness of Cauchy-type integrals, Ukrainian Math. J. 29, no. 5 (1977), 490-493.

[4] Gerus, O. F., Some estimates of moduli of smoothness of integrals of the Cauchy type, Ukrainian Math. J. 30, no. 5 (1978), 594-601.

[5] Ketchum, P. W., Analytic functions of hypercomplex variables, Trans. Amer. Math. Soc. 30 (1928), 641-667.

[6] Kunz, K. S., Application of an algebraic technique to the solution of Laplace's equation in three dimensions, SIAM J. Appl. Math. 21, no. 3 (1971), 425-441.

[7] Mel'nichenko, I. P., The representation of harmonic mappings by monogenic functions, Ukrainian Math. J. 27, no. 5 (1975), 499-505.

[8] Mel'nichenko, I. P., Algebras of functionally invariant solutions of the threedimensional Laplace equation, Ukrainian Math. J. 55, no. 9 (2003), 1551-1559.

[9] Mel'nichenko, I. P., Plaksa, S. A., Commutative algebras and spatial potential fields, Inst. Math. NAS Ukraine, Kiev, 2008 (Russian).

[10] Plaksa, S. A., Riemann boundary-value problem with infinite index of logarithmic order on a spiral contour. I, Ukrainian Math. J. 42, no. 11 (1990), 1509-1517. 
[11] Shpakivskyi, V. S., Plaksa, S. A., Integral theorems and a Cauchy formula in a commutative three-dimensional harmonic algebra, Bull. Soc. Sci. Lett. Łódź Sér. Rech. Déform. 60 (2010), 47-54.

S. A. Plaksa

Department of Complex Analysis and Potential Theory

Institute of Mathematics of the National Academy of Sciences of Ukraine

Tereshchenkivska St. 3

$01601 \mathrm{Kiev}-4$

Ukraine

e-mail: plaksa@imath.kiev.ua

V. S. Shpakivskyi

Department of Complex Analysis and Potential Theory

Institute of Mathematics of the National Academy of Sciences of Ukraine

Tereshchenkivska St. 3

01601 Kiev-4

Ukraine

e-mail: shpakivskyi@mail.ru

Received September 30, 2011 